\title{
ABUNDANCES IN EVOLVED INTERMEDIATE MASS STARS
}

\author{
M. PERINOTTO \\ Dipartimento di Astronomia, Università di Firenze, Italy \\ AND \\ L. MORBIDELLI AND P. PATRIARCHI \\ Consiglio Nazionale delle Ricerche, CAISMI, Firenze, Italy
}

A sample of 285 galactic Planetary Nebulae (PNe) well studied up to 1995 has been examined to improve our knowledge on Galactocentric Chemical Gradients (GCG) and chemical enrichment of the Interstellar Medium (ISM). The sample has been subdivided following Peimbert (1978). We have examined the vertical gradients of $\mathrm{PNe}$ within a cylinder normal to the galactic plane with axis on the Sun and radius of $2 \mathrm{kpc}$. Excluding PNe of type $I V$ we don't see vertical gradients. The spread of $\log (O / H)$ near the galactic plane for type $I I \mathrm{PNe}$ is of 0.7 dex.This is larger than the maximum uncertainty of the individual determination of the abundance of oxygen, estimated to be of $\pm 50 \%( \pm 0.18 \mathrm{dex})$. This supports the existence of chemical inhomogeneites in the ISM in the last $0.5 \div 5 \mathrm{Gyr}$. The above allows to look for GCG projected on the galactic plane. For PNe of type $I I$, GCG in usual units amount to $+0.002 \pm 0.02,+0.024 \pm 0.03$, $-0.075 \pm 0.15,-0.027 \pm 0.07,-0.044 \pm 0.10,-0.011 \pm 0.15,-0.067 \pm 0.10$ for $\mathrm{He}, \mathrm{C}, \mathrm{N}, \mathrm{O}, \mathrm{Ne}, \mathrm{S}, \mathrm{Ar}$ respectively. The corresponding values from FaundezAbans \& Maciel (1987) are $-0.019 \pm 0.024,-0.069 \pm 0.017,-0.072 \pm 0.24$, $-0.072 \pm 0.10,-0.056 \pm 0.15,-0.098 \pm 0.18,-0.056 \pm 0.15$ Note that our gradient for oxygen is quite different but within the uncertainties. We estimate the ISM chemical enrichment from PNe (Tinsley, 1978). For type $I$ PNe we find: $H e(P N) / H e_{\odot}=1.4, N(P N) / N_{\odot}=4.1$. The relative mass return to the $I S M$ from $\mathrm{PNe}$ comes out to be four times larger than from type $I I \mathrm{SN}$, with a possible uncertainty of a factor $2 \div 3$.

\section{References}

Faundez-Abans, M. and Maciel W.J. (1987) Astrophys. Spa. Sci,129, 353

Peimbert, M. (1978) IAU Symp. 76: Planetary Nebulae, ed. Y. Terzian

Tinsley, B.M. (1978) IAU Symp. 76: Planetary Nebulae, ed. Y. Terzian, p. 341 